\title{
Intrinsic iron spread and a new metallicity scale for globular clusters ${ }^{\star}$
}

\author{
E. Carretta ${ }^{1}$, A. Bragaglia ${ }^{1}$, R. Gratton ${ }^{2}$, V. D’Orazi ${ }^{2}$, and S. Lucatello ${ }^{2,3}$ \\ 1 INAF-Osservatorio Astronomico di Bologna, via Ranzani 1, 40127 Bologna, Italy \\ e-mail: eugenio.carretta@oabo.inaf.it \\ 2 INAF-Osservatorio Astronomico di Padova, Vicolo dell'Osservatorio 5, 35122 Padova, Italy \\ 3 Excellence Cluster Universe, Technische Universität München, Boltzmannstr. 2, 85748 Garching, Germany
}

Received 28 July 2009 / Accepted 1 October 2009

\section{ABSTRACT}

\begin{abstract}
We have collected spectra of about 2000 red giant branch (RGB) stars in 19 Galactic globular clusters (GC) using FLAMES@VLT (about 100 stars with GIRAFFE and about 10 with UVES, respectively, in each GC). These observations provide an unprecedented, precise, and homogeneous data-set of Fe abundances in GCs. We use it to study the cosmic scatter of iron and find that, as far as $\mathrm{Fe}$ is concerned, most GCs can still be considered mono-metallic, since the upper limit to the scatter of iron is less than 0.05 dex, meaning that the degree of homogeneity is better than $12 \%$. The scatter in Fe we find seems to have a dependence on luminosity, possibly due to the well-known inadequacies of stellar atmospheres for upper-RGB stars and/or to intrinsic variability. It also seems to be correlated with cluster properties, like the mass, indicating a larger scatter in more massive GCs which is likely a (small) true intrinsic scatter. The $19 \mathrm{GCs}$, covering the metallicity range of the bulk of Galactic GCs, define an accurate and updated metallicity scale. We provide transformation equations for a few existing scales. We also provide new values of $[\mathrm{Fe} / \mathrm{H}]$, on our scale, for all GCs in the Harris catalogue.
\end{abstract}

Key words. stars: abundances - stars: atmospheres - stars: population II - globular clusters: general

\section{Introduction}

The chemical composition of globular cluster (GC) stars cannot be regarded anymore as strictly homogeneous, as implied from the classical paradigm that these old stellar aggregates were the best approximation in nature of simple stellar populations (see Gratton et al. 2004, for an extensive review and references). Recent investigations using large samples of stars observed with multi-object spectrographs at large telescopes highlight that every GC studied so far harbours at least two different stellar generations, distinct in chemical composition and age (Carretta et al. 2009a,b).

These different populations are found to differ in abundances of light elements (C, N, O, Na, Mg, Al, Si, F; Smith \& Martell 2003; Smith et al. 2005; Carretta et al. 2009a,b; Yong et al. 2008a,b; Melendez \& Cohen 2009, to quote a few studies; see also Gratton et al. 2004, and references therein) involved in proton-capture reactions of H-burning at high temperature (Denisenkov \& Denisenkova 1989; Langer et al. 1993). Star to star abundance variations in these elements are expected to also come with differences in the main outcome of the $\mathrm{H}$ burning, the He content (Gratton et al. 2009; Bragaglia et al. in preparation; Prantzos \& Charbonnel 2006; Ventura et al. 2001). Apart from a few alterations presently well understood in term of an extra-mixing episode after the red giant branch (RGB) bump (Charbonnel 1994, 1995; Charbonnel \& Zahn 2007; Eggleton et al. 2007) the abundance variations are inherited by currently observed, long lived GC stars from a previous stellar component/generation: both spectroscopic (Gratton et al. 2001;

^ Based on observations collected at ESO telescopes under programmes 072.D-507 and 073.D-0211.
Ramirez \& Cohen 2002; Carretta et al. 2004; Piotto et al. 2005) and photometric (e.g. Bedin et al. 2004) observations convincingly showed that the observed pattern of chemical composition is present also among unevolved stars on the subgiant branch and the main sequence.

On the other hand, apart from a few notable exceptions ${ }^{1}$ GCs are still found to be mono-metallic objects, as far as abundances of heavier elements are concerned (see Gratton et al. 2004, for a recent review on this subject). Their heavy $(Z>13)$ element metallicity, usually represented by the ratio $[\mathrm{Fe} / \mathrm{H}]^{2}$, is found to be extremely homogeneous from star to star in each cluster.

The sites of production of heavy elements (in particular $\alpha$ capture elements, Fe-group elements) are stars with large and intermediate initial masses, exploding as core-collapse or thermonuclear supernovae (see Wheeler et al. 1989). By studying the level and the dispersion of their yields inherited by stars in GCs we have the possibility of investigating the past history of the precursors from whom the present-day globular clusters formed (see Carretta et al. 2009c).

The recently completed analysis of high resolution spectra of almost 2000 RGB stars in 19 Galactic GCs (Carretta et al. 2009a,b and references therein) provides an unprecedented

1 The GC $\omega$ Cen (widely considered to be the remnant of a dwarf galaxy) shows evidence of several bursts of star formation, with corresponding peaks in the metal abundance (see Gratton et al. 2004 for a comprehensive discussion) and a spread in metallicity seems to be confirmed in M 22 (Marino et al. 2009) as well as in M 54 (Bellazzini et al. 2008; Carretta et al., in preparation).

${ }^{2}$ We adopt the usual spectroscopic notation, i.e. $[X]=\log (X)_{\text {star }}-$ $\log (X)_{\odot}$ for any abundance quantity $X$, and $\log \epsilon(X)=\log \left(N_{\mathrm{X}} / N_{\mathrm{H}}\right)+$ 12.0 for absolute number density abundances. 
sample of stars analysed in a fully homogeneous way. By exploiting these data we can examine in detail the issue of the cosmic scatter in the metallicity (hereafter the abundance of Fe-peak elements) of GCs and hopefully provide clues to the early evolution of their progenitors.

Moreover, abundances of iron from high resolution spectra are traditionally the calibrating points for metallicity scales based on photometric and/or low-resolution spectroscopic indices that are sensitive to metal abundances (see the excellent discussion and historical review on this issue by Kraft \& Ivans 2003). Thus, our sample is perfectly suited to provide robust (for statistics) and homogeneous (for technique of analysis) "pillars" onto which anchor several existing metallicity scales.

The paper is organized as follows. We give in Sect. 2 a brief description of the data set and of the analysis. We discuss the intrinsic (cosmic) spread of iron in Sect. 3, where we also present some correlations of the spread with GC properties, like the absolute total luminosity or the $\alpha$-elements abundance. We discuss the dependence of this spread on the stars luminosity in Sect. 4 and present a recalibration of four metallicity scales to our new one in Sect. 5. A summary is given in Sect. 6 and we give metallicity values on our new scale for all GCs in the Harris (1996) catalogue in the Appendix.

\section{The dataset and analysis}

Briefly, in our survey we collected GIRAFFE spectra of intermediate resolution $(R \simeq 20000)$ for about 100 stars and UVES spectra $(R \simeq 40000)$ for about 10 stars, on average, in each of our 19 programme GCs. The GIRAFFE gratings were chosen to include the $\mathrm{Na}$ I lines at 568.2-568.8 $\mathrm{nm}$ (grating HR11) and the forbidden [O I] lines at $630.0-636.3 \mathrm{~nm}$ (HR13). The number of clean Fe I lines typically falling in the corresponding spectral ranges, and used in our analysis, varied from about 10 to a maximum of about 40, depending on the $S / N$, the metallicity and whether the stars were observed with HR11 only, HR13 only, or both. The UVES spectra covered the 480-680 nm region and plenty of $\mathrm{Fe}$ lines were measured even in the most metal-poor clusters.

The data analysis has already been described in detail elsewhere (Carretta et al. 2006, 2007a-c; Gratton et al. 2006, 2007; Carretta et al. 2009a,b) and will not be repeated here. Only a few procedures will be briefly summarised here. Atmospheric parameters were homogeneously derived for all stars using visual and near-IR photometry and the relations given by Alonso et al. (1999, 2001). Equivalent widths (EWs) were measured by an updated semi-automatic routine, in the package ROSA (Gratton 1988), using an homogeneous method for the continuum positioning (described in Bragaglia et al. 2001). The line list is adopted from Gratton et al. (2003), where the atomic parameters and solar reference abundances are fully discussed. This list was used for all stars in our program clusters, and we believe that this represents a remarkable strength of this project, resulting in a huge database of stellar abundances derived in the most homogeneous way for a significant part $(\sim 12 \%)$ of the Galactic GC population, spanning the range in $[\mathrm{Fe} / \mathrm{H}]$ from -2.5 to -0.4 dex.

As discussed in the papers quoted above, all $E W$ s measured on the GIRAFFE spectra were shifted to a system defined by those derived from high resolution UVES spectra, using stars and lines observed with both instruments.

Taking into account the stars in common between UVES and GIRAFFE observations, we have values of $[\mathrm{Fe} / \mathrm{H}]$ for 1958 individual RGB stars in 19 GCs. Average abundances of iron derived from both UVES and GIRAFFE spectra are summarised in Table 1. Only abundances derived from neutral Fe lines are reported (and will be used in the following) because we already showed in Papers VII and VIII that the differences in iron abundances as obtained from neutral and singly ionised species are negligible in our analysis ${ }^{3}$. Thus, we will adopt the $[\mathrm{Fe} / \mathrm{H}]$ I ratio as representative of the metallicity of each star, because it is based on a much larger number of lines, thus providing a more robust estimate.

Once $E W \mathrm{~s}$ measured from GIRAFFE spectra are corrected to the system defined by UVES spectra, the abundance analysis provides very similar results for the average metallicity in each cluster: the mean difference (in the sense UVES minus GIRAFFE) is $-0.015 \pm 0.008$ dex with an rms scatter of 0.037 dex from 19 GCs. The largest difference $(0.098 \mathrm{dex})$ is found for NGC 6441, for which the observations in service mode could not be completed as requested, so that the available spectra have low $S / N$, due both to the large distance modulus and incomplete observations. Figure 1, reproduced from Paper VIII, shows the quite good agreement between abundances of iron as obtained from both instruments; thus, although we will use $[\mathrm{Fe} / \mathrm{H}]$ I values from the high resolution UVES spectra, we are confident that the new metallicity scale presented in the present paper rests ultimately on very accurate and homogeneous abundances for a sample of almost 2000 cluster stars.

\section{Intrinsic spread of iron in globular clusters}

We compare in Fig. 2 the bona fide observed intrinsic spreads in $\mathrm{Fe}$ (defined as the rms scatter of all stars in each cluster), as given by GIRAFFE and UVES spectra (Cols. 5 and 8 in Table 1). The error bars show the maximum and minimum spread allowed for each cluster taking into account the statistical errors.

Looking at this figure, two features are immediately evident: first, on average, the rms scatter obtained from UVES spectra is larger than the one we derived from the analysis of GIRAFFE spectra. At first sight, this result is just the opposite one would expect, given the higher resolution and spectral coverage of the UVES spectra. Possible explanations for this effect will be examined in the next section.

Second, intrinsic scatters from GIRAFFE spectra show quite small associated error bars, owing to the much larger statistics; if we focus on these values, the first conclusion is that the cosmic scatter in Fe in globular clusters is very small. On average, we found a value of $0.048 \mathrm{dex}$ (with $\sigma=0.018 \mathrm{dex}$ ) from $19 \mathrm{GCs}$; i.e. the iron abundance in each cluster is homogeneous within $12 \%$.

Furthermore, we note that these are strictly upper limits to the actual dispersion of $[\mathrm{Fe} / \mathrm{H}]$ values in GCs. The true intrinsic values should be estimated by subtracting (in quadrature) the scatter expected from errors in the analysis, namely in the derivation of the atmospheric parameters and in the measurement of $E W s$. These quantities were estimated with a thorough procedure amply described in previous papers and are reported in the Appendix of Paper VII and in Papers I to VI. Unfortunately, for the GIRAFFE spectra these estimates often exceed the observed scatter, probably because of the overestimate of the effect of some error sources, making difficult to derive the intrinsic scatter by deconvolving for this uncertainty. For 8 out 19 GCs this deconvolution was possible and from this, as well as from the analysis of UVES spectra, we found that a reasonable estimate is that the observed values of the rms scatter must be

\footnotetext{
3 Note that we usually avoided observations of stars close to the RGB tip, that may be more problematic.
} 
Table 1. Average abundances of $[\mathrm{Fe} / \mathrm{H}] \mathrm{I}$ and observed rms scatters in our sample.

\begin{tabular}{|c|c|c|c|c|c|c|c|c|c|}
\hline$\overline{\mathrm{GC}}$ & $\begin{array}{l}\text { alt. } \\
\text { name }\end{array}$ & $\begin{array}{r}\text { nr. } \\
\text { stars } \\
\text { GIRAFFE }\end{array}$ & $\begin{array}{l}{[\mathrm{Fe} / \mathrm{H}] \mathrm{I}} \\
\pm \text { stat.err } \pm \text { syst. } \\
(\mathrm{dex})\end{array}$ & rms & $\begin{array}{r}\mathrm{nr} . \\
\text { stars } \\
\text { UVES }\end{array}$ & $\begin{array}{l}{[\mathrm{Fe} / \mathrm{H}] \mathrm{I}} \\
\pm \text { stat.err } \pm \text { syst. } \\
(\mathrm{dex})\end{array}$ & rms & GIRAFFE & UVES \\
\hline NGC 104 & $47 \mathrm{~T}$ & 147 & $-0.743 \pm 0.003 \pm 0.026$ & 0.032 & 11 & $-0.768 \pm 0.016 \pm 0.031$ & 0.054 & Paper VII & Paper VIII \\
\hline NGC 288 & & 110 & $004 \pm 0.070$ & 0.042 & 10 & -1 . & 0. & VII & aper VIII \\
\hline NGC 1904 & M 79 & 58 & $.005 \pm 0.069$ & 0.036 & 10 & -1.579 & 0.033 & Paper VII & Paper VIII \\
\hline NGC 2808 & & 123 & $-1.104 \pm 0.006 \pm 0.046$ & 0.065 & 12 & $-1.151=$ & 0.075 & Paper I & Paper VIII \\
\hline NGC 3201 & & 149 & $-1.495 \pm 0.004 \pm 0.073$ & 0.049 & 13 & $-1.512 \pm 0.0$ & 0.065 & Paper VII & Paper VIII \\
\hline NGC 4590 & M 68 & 122 & $-2.227 \pm 0.006 \pm 0.068$ & 0.071 & 13 & $-2.265 \pm 0.013 \pm 0.070$ & 0.047 & Paper VII & Paper VIII \\
\hline NGC 5904 & M 5 & 136 & $-1.346 \pm 0.002 \pm 0.062$ & 0.023 & 14 & $-1.340 \pm 0.014 \pm 0.064$ & 0.052 & Paper VII & Paper VIII \\
\hline NGC 6121 & M 4 & 103 & $-1.200 \pm 0.002 \pm 0.053$ & 0.025 & 14 & $-1.168 \pm 0$. & 0.046 & aper VII & Paper VIII \\
\hline NGC 6171 & M 107 & 33 & $-1.065 \pm$ & 0.044 & 5 & $-1.033=$ & 0.064 & er VII & Paper VIII \\
\hline NGC 6218 & M 12 & 79 & -1. & 0.0 & 11 & -1 . & 0.042 & IV & Paper VIII \\
\hline NGC & M 10 & 147 & & 0.053 & 14 & -1 & 0.059 & Paper VII & Paper VIII \\
\hline NGC 6388 & & 36 & -0 . & 0.078 & 7 & -0 & 0.038 & Paper VII & Paper VI \\
\hline NGC 6397 & & 144 & $-1.993 \pm 0.003 \pm 0.060$ & 0.039 & 13 & & 0.044 & Paper VII & Paper VIII \\
\hline NGC 6441 & & 25 & & 0.087 & 5 & & 0.058 & aper V & aper III \\
\hline NGC 6752 & & 37 & -1 & & 14 & & 0.051 & & per VIII \\
\hline & M 55 & 156 & -1 & & 14 & & 0.063 & VII & Paper VIII \\
\hline & M 71 & 39 & -0.8 & & 12 & & 0.061 & VII & Paper VIII \\
\hline 7078 & M 15 & 84 & -2 . & 0. & 13 & -2 & 0.057 & Paper VII & Paper VIII \\
\hline NGC 7099 & M 30 & 64 & $-2.359 \pm 0.006 \pm 0.067$ & 0.046 & 10 & $-2.344 \pm 0.015 \pm 0.069$ & 0.049 & Paper VII & Paper VIII \\
\hline
\end{tabular}

Columns 9 and 10 provide the references of the papers where the analysis of all GIRAFFE and UVES data, respectively, is presented for each cluster.

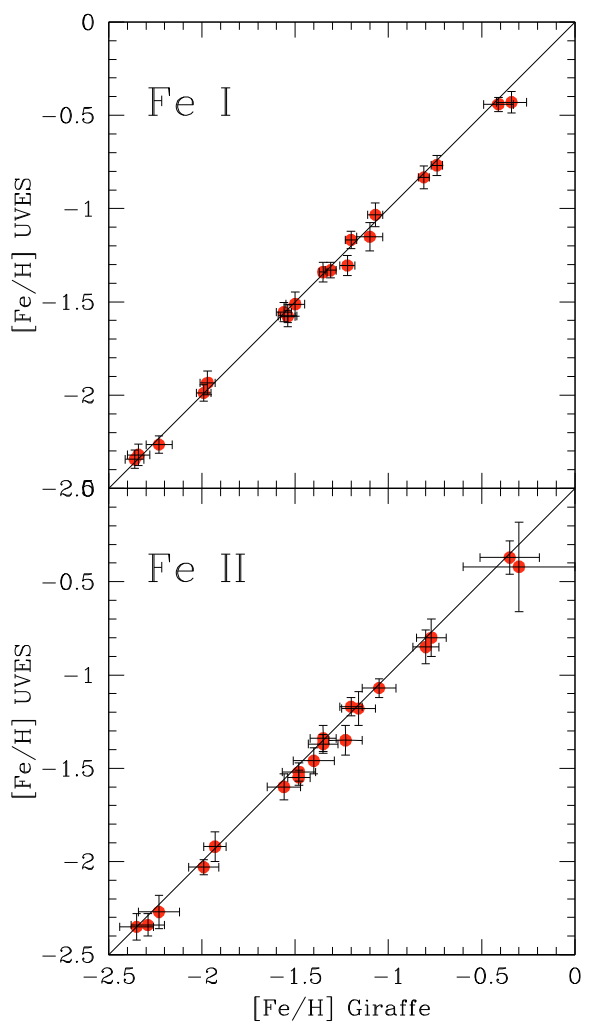

Fig. 1. Comparison for [Fe/H] I ratios (upper panel) and for [Fe/H] II ratios (lower panel) obtained from GIRAFFE and UVES spectra in the 19 programme clusters in our sample. Metal abundances from GIRAFFE are from Papers I, II, IV, V, and VII. Metallicities from UVES are from Paper III, VI, and VIII. Error bars are $1 \sigma$ rms scatter and the solid line is the line of equality.

further reduced by $1-2 \%$, to account for the analysis uncertainties.

To obtain a larger statistical sample we considered the observed scatters, noting however that they are upper limits.

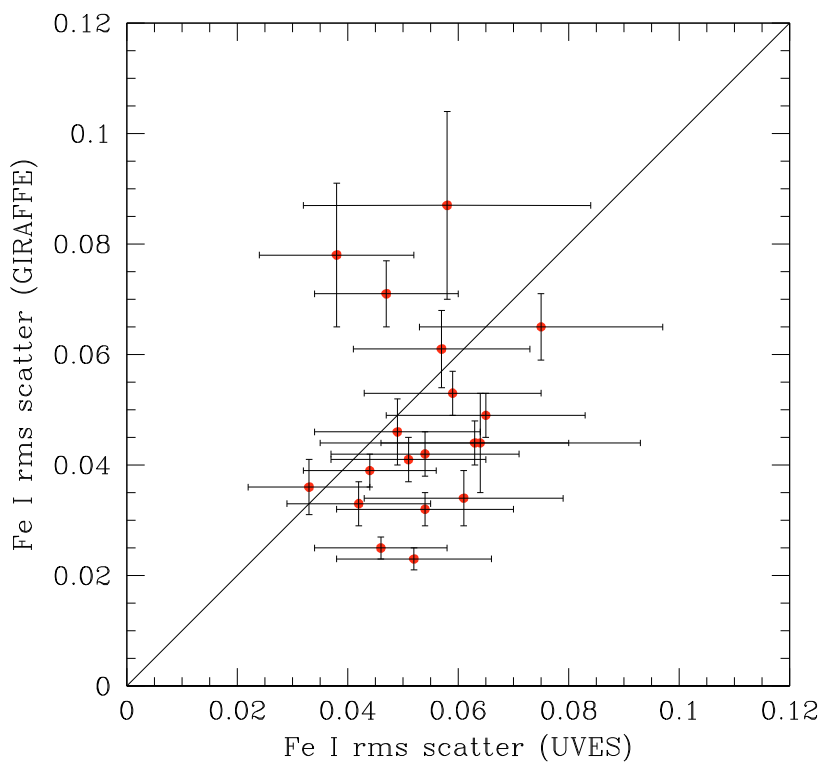

Fig. 2. Comparison of the intrinsic spreads in $[\mathrm{Fe} / \mathrm{H}]$ for the $19 \mathrm{GCs}$ in our sample, as derived from UVES and GIRAFFE spectra. Error bars show the maximum and minimum spread allowed for each cluster by the statistical errors. The line indicating equality is also shown.

Therefore, our first conclusion is that, as far as iron is concerned, at first approximation the GCs can still be considered monometallic aggregates, apart from the few exceptions mentioned in the Introduction.

However, the high degree of homogeneity in our analysis, coupled with our very large sample, allow us to highlight subtle features that might be lost in the noise when collecting data from separate sources. In particular, we found several statistically significant relations between the intrinsic iron spread and global cluster parameters. In Table 2 we summarise the Pearson's linear correlation coefficients, the degrees of freedom and the statistical significance for a number of these relations. 
Table 2. Pearson's correlation coefficients, degrees of freedom and significance for relations with iron spread in our GC sample.

\begin{tabular}{llll}
\hline \hline Parameter & $r_{\mathrm{p}}$ & d.o.f. & signif. \\
\hline$M_{V}$ & -0.49 & 17 & $95-98 \%$ \\
$R_{\text {perig. }}$ & +0.47 & 15 & $90-95 \%$ \\
$\log T_{\text {eff }}^{\max }(\mathrm{HB})$ & +0.38 & 17 & $90 \%$ \\
$x(\mathrm{MF})$ & +0.60 & 11 & $95-98 \%$ \\
rel.Age & -0.49 & 17 & $95-98 \%$ \\
$\sigma(\mathrm{h})$ & +0.59 & 16 & $99 \%$ \\
$\mathrm{P}$ & +0.65 & 17 & $>99 \%$ \\
$\mathrm{I}$ & -0.67 & 17 & $>99 \%$ \\
$\mathrm{E}$ & +0.52 & 17 & $\sim 98 \%$ \\
{$[(\mathrm{Mg}+\mathrm{Al}+\mathrm{Si}) / \mathrm{Fe}]$} & -0.48 & 16 & $95-98 \%$ \\
{$[\langle\mathrm{Si}, \mathrm{Ca}\rangle / \mathrm{Fe}]$} & -0.70 & 17 & $>99 \%$ \\
{$[\mathrm{Ca} / \mathrm{Fe}]$} & -0.78 & 17 & $>99 \%$ \\
$[<\alpha>] / \mathrm{Fe}]$ & -0.72 & 17 & $>99 \%$ \\
{$[\mathrm{Mg} / \mathrm{Fe}]_{\max }$} & -0.52 & 17 & $98 \%$ \\
{$[\mathrm{Mg} / \mathrm{Fe}]_{\min }$} & -0.56 & 17 & $98-99 \%$ \\
{$[\mathrm{Si} / \mathrm{Fe}]_{\min }$} & -0.44 & 17 & $90-95 \%$ \\
\hline
\end{tabular}

$-M_{V}$ from the on-line version of the Harris (1996) catalogue.

- Peri-galactic distance $R_{\text {perig. }}$ in kpc from Dinescu et al. (1999) or Casetti-Dinescu et al. (2007).

- Maximum temperature along the HB $\log T^{\max }(\mathrm{HB})$ from Recio Blanco et al. (2006) or derived in Carretta et al. (2009c).

- Exponent $x$ of the power-law relation for global mass function from Djorgovski et al. (1993).

- Relative age parameter from De Angeli et al. (2005) and Rosenberg et al. (1999).

- Velocity dispersion at the half-mass radius $\sigma(h)$ from Gnedin et al. (2002).

- Fractions of primordial $P$, intermediate $I$ and extreme $E$ components from Carretta et al. (2009a; Paper VII).

- The other abundance ratios are derived from UVES spectra (Carretta et al. 2009b; Paper VIII); here $[\alpha / \mathrm{Fe}]$ is the average of $[\mathrm{Mg} / \mathrm{Fe}]_{\max }$, $[\mathrm{Si} / \mathrm{Fe}]_{\min }$ and $[\mathrm{Ca} / \mathrm{Fe}]$.

Our main finding is that the observed dispersion in iron is correlated with the cluster absolute magnitude $M_{V}$ (upper panel in Fig. 3), a proxy for the cluster present-day mass. More massive clusters show a larger spread in $[\mathrm{Fe} / \mathrm{H}]$. Note that this correlation would extend to brighter cluster absolute magnitudes if we include very massive clusters like M 54 and $\omega$ Cen.

The rms seems preferentially larger in farther GCs, which could possibly indicate that the trend may be driven by the data quality. However, this is unlikely to be an observational artifact; as shown in the middle panel of Fig. 3, where we plot the median $S / N$ ratio for the HR13 spectra, there is not a significant correlation between the quality of spectra and the rms scatter. Most clusters have a typical $S / N$ around 120 , yet show very different observed scatters. Moreover, our sample includes disk and inner halo GCs, and in general more massive clusters are preferentially found at larger distances (i.e. among the inner halo component) also in the global population of galactic GCs (Harris 1996).

Several theoretical models (Larson 1987; Suntzeff \& Kraft 1996; D'Ercole et al. 2008; Decressin et al. 2009) suggest that currently observed GCs are only a small fraction in mass of the original parent structures where they formed. In Carretta et al. (2009c) we proposed a formation scenario for GCs where a socalled precursor raises the metallicity to a level which corresponds to the primordial abundances currently observed in a cluster. In other words, in present-day GCs we are not seeing the contribution of massive SNe: the level of iron and $\alpha$-elements is already homogeneously established in the gas by the explosion of core-collapse $\mathrm{SNe}$ in the precursor/proto-cluster. Of course the idea of pre-enrichment in large fragments seeding the present
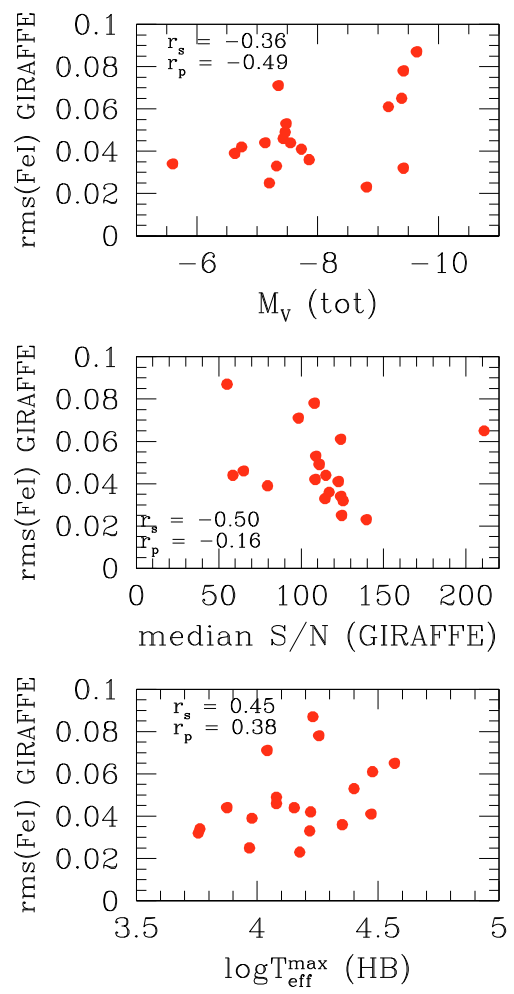

Fig. 3. Observed intrinsic dispersion in iron from GIRAFFE spectra as a function of the visual total absolute magnitude of the clusters (upper panel), of the median $S / N$ for HR13 spectra (middle panel), and of the maximum temperature along the horizontal branch (lower panel). In each panel of this and the next figures we list the Spearman and Pearson correlation coefficients.

galactic GC population is not new (see Searle \& Zinn 1978). In our scenario, the second effect of $\mathrm{SNe}$ in the precursor is to trigger a burst of star formation leading to the build up of the primordial population, a fraction of which we can still observe in GCs, making up about $1 / 3$ of the total cluster stars (see Carretta et al. 2009a). However, the correlation we see between iron spread and cluster mass tells us that clusters originating in more massive precursors are probably more capable to retain inhomogeneities in the metallicity plateau established in the previous phase of rapid enrichment.

This is just what is observed for the rms scatter in $[\mathrm{Fe} / \mathrm{H}]$ as a function of $M_{V}$ and of other cluster parameters strictly related to the cluster mass: the maximum temperature reached along the cluster HB (lower panel of Fig. 3; see Recio Blanco et al. 2006; Carretta et al. 2009c), the slope of the cluster global mass function (see Djorgovski et al. 2003), the velocity dispersion at halfmass radius (Gnedin et al. 2002).

We note that a (small) part of the higher values for the spread in more massive clusters may also be due to variations in He content, which are expected to be larger in GCs of larger masses (Gratton et al. 2009). In this case, of course, the effect is due not only to the higher capability to retain ejecta but also to the enhanced He in some stars formed by gas heavily polluted in $\mathrm{H}$-processed material, which lowers the denominator in the $[\mathrm{Fe} / \mathrm{H}]$ ratio (see also Bragaglia et al. in prep.). We can check the relevance of this effect by computing the iron spread using only stars in the primordial component $P$ (see Carretta et al. 2009a), which are not expected to be formed from matter polluted with additional He. 

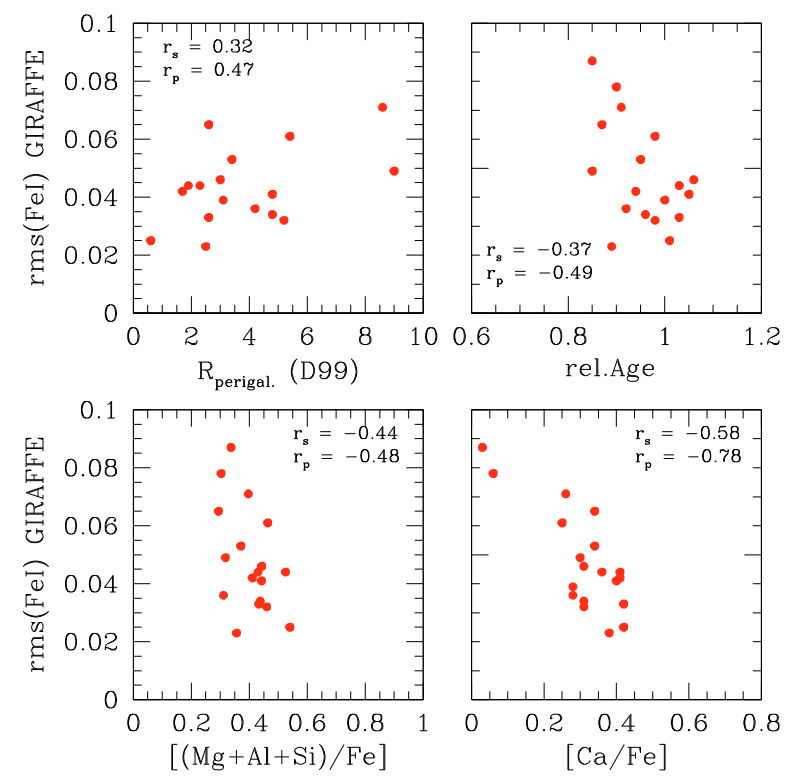

Fig. 4. Observed spread of $[\mathrm{Fe} / \mathrm{H}]$ in our programme clusters as a function of their peri-galactic distance (upper left panel), age (upper right panel), total sum of $\mathrm{Mg}+\mathrm{Al}+\mathrm{Si}$ nuclei (lower left panel) and $[\mathrm{Ca} / \mathrm{Fe}]$ ratio.

We found that the difference in the rms of $[\mathrm{Fe} / \mathrm{H}]$ (in the sense all stars minus $P$ stars) is on average $-0.002 \pm 0.003$ (with $\sigma=0.012$ for $19 \mathrm{GCs}$ ). For the two most massive clusters in the sample (NGC 6388 and NGC 6441) this difference goes in opposite directions and the largest differences are found for the three GCs with lower quality spectra (NGC 6388 and NGC 6441 again, plus NGC 6752). We conclude that the contribution to the spread in iron caused by variations in the He content is probably very small.

A support for the proposed scenario comes from a second set of (anti)correlations involving the observed spread of iron and the position of GCs in the Galaxy (or related quantities; see Table 2 and Fig. 4). The dispersion in $[\mathrm{Fe} / \mathrm{H}]$ is larger in GCs spending, on average, more time at larger distances from the Galactic centre (upper left panel). At the same time, the spread decreases for older clusters and for increasing abundances of the $\alpha$-element ${ }^{4}$.

In Carretta et al. (2009c) we emphasised that inner halo clusters preferentially include more massive clusters with respect to disc clusters; moreover, the first are also younger, on average, and with a slightly smaller level of $\alpha$-elements than that found in disc/bulge GCs. All these features concur to explain the good correlation and the anti-correlations displayed in Fig. 4.

Finally, in Fig. 5 we show the run of the rms scatter in Fe as a function of the fraction of first generation stars $P$, as well as the second generation components of intermediate modified $(I)$, and extreme modified $(E)$ composition (see Carretta et al. 2009a for detailed definitions of these stellar populations). The dispersion in Fe increases along with the fraction of $P$ stars still observed in GCs, again pointing toward a deeper potential well in more massive clusters, more able to retain both SN ejecta and low mass $P$ stars. On the other hand, we know that the fraction of $E$ stars is larger in more massive clusters, and this explains also the correlation between the spread and the $E$ component.

${ }^{4}$ The $[(\mathrm{Mg}+\mathrm{Al}+\mathrm{Si}) / \mathrm{Fe}]$ ratio represents the primordial abundances of $\alpha$-elements in a cluster, mostly $\mathrm{Mg}$ and $\mathrm{Si}$, as modified by the following conversion of $\mathrm{Mg}$ into $\mathrm{Al}$ and, slightly, into $\mathrm{Si}$ in $\mathrm{H}$-burning at high temperature (see Carretta et al. 2009b).

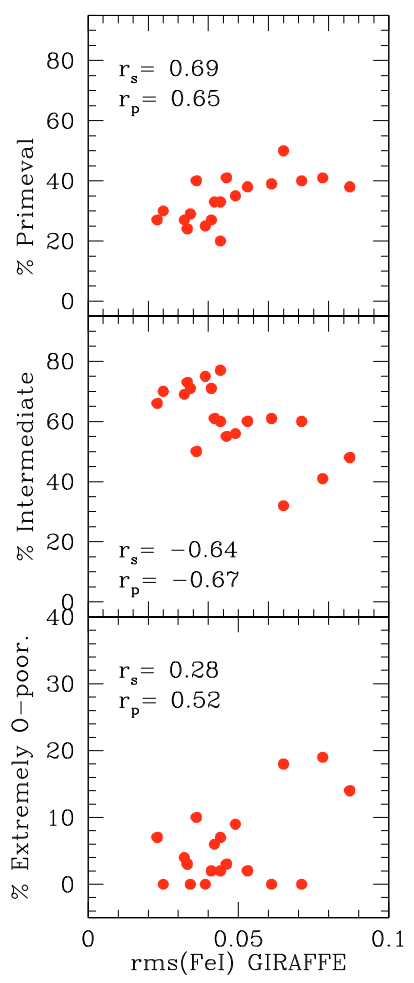

Fig. 5. Fraction of stars in the $P$ (upper panel), $I$ (middle panel), and $E$ component (lower panel) of our programme clusters as a function of the observed rms scatter in $[\mathrm{Fe} / \mathrm{H}]$.

The complementarity between $I$ and $E$ fractions (Carretta et al. 2009c) well accounts for the anti-correlation shown in the middle panel of Fig. 5.

\section{Fe scatter as a function of the luminosity}

Coming back to the comparison of the rms scatters in Table 1, we now look at the rather surprising fact that the rms we derive for Fe abundances is higher from the high-resolution UVES spectra than from the intermediate-resolution GIRAFFE ones. This effect is particularly clear for clusters with metallicity $-1.4<$ $[\mathrm{Fe} / \mathrm{H}]<-0.7$ dex in our programme sample, which have data of higher quality. More metal-rich clusters (e.g. NGC 6388 and NGC 6441) are quite distant, bulge clusters, severely affected by field contamination and required a series of long exposures which were not always completed in the ESO observing service queue (in particular for NGC 6441). Analysis of more metalpoor clusters is somewhat hampered by the weakness of lines, increasing the difficulty of $E W$ measurements.

To explain the abovementioned effect, a possibility is that stars observed with UVES have intrinsically a larger scatter than those observed with GIRAFFE. This would happen if the intrinsic scatter is partly a function of the stellar luminosity, because we preferentially chose brighter stars as UVES targets (although the majority of the objects - about 170 out of 214 stars - are in common between the two data sets).

To better understand this point we need to improve the estimate of the intrinsic scatter and in order to clarify the issue we proceeded as follows. For each star observed with GIRAFFE or UVES (separately) we evaluated the difference between the $[\mathrm{Fe} / \mathrm{H}]$ value for the individual stars and the average value $[\mathrm{Fe} / \mathrm{H}]$ of the cluster. These residuals were then plotted as a function of the absolute $K$ magnitude $M_{K}^{0}$ corrected for the apparent distance 
Table 3. Average residuals with respect to the mean $[\mathrm{Fe} / \mathrm{H}]$ for clusters with metallicities $-1.4<[\mathrm{Fe} / \mathrm{H}]<-0.7$ dex.

\begin{tabular}{rrcc}
\hline \hline$M_{K}^{0}$ bin & stars & $\left\langle[\mathrm{Fe} / \mathrm{H}]_{\mathrm{star}}-[\mathrm{Fe} / \mathrm{H}]_{\mathrm{GC}}\right\rangle$ & $\mathrm{rms}$ \\
\hline$+0.0 \div-1.0$ & 97 & GIRAFFE \\
$-1.0 \div-2.0$ & 271 & $+0.006 \pm 0.004$ & 0.035 \\
$-2.0 \div-3.0$ & 155 & $-0.009 \pm 0.002$ & 0.033 \\
$-3.0 \div-4.0$ & 81 & $+0.004 \pm 0.006$ & 0.038 \\
$-4.0 \div-5.0$ & 56 & $-0.012 \pm 0.006$ & 0.065 \\
& UVES \\
$-1.5 \div-3.0$ & 16 & $+0.008 \pm 0.014$ & 0.056 \\
$-3.0 \div-4.0$ & 15 & $-0.004 \pm 0.008$ & 0.051 \\
$-4.0 \div-5.5$ & 30 & $-0.001 \pm 0.010$ & 0.058 \\
\hline
\end{tabular}

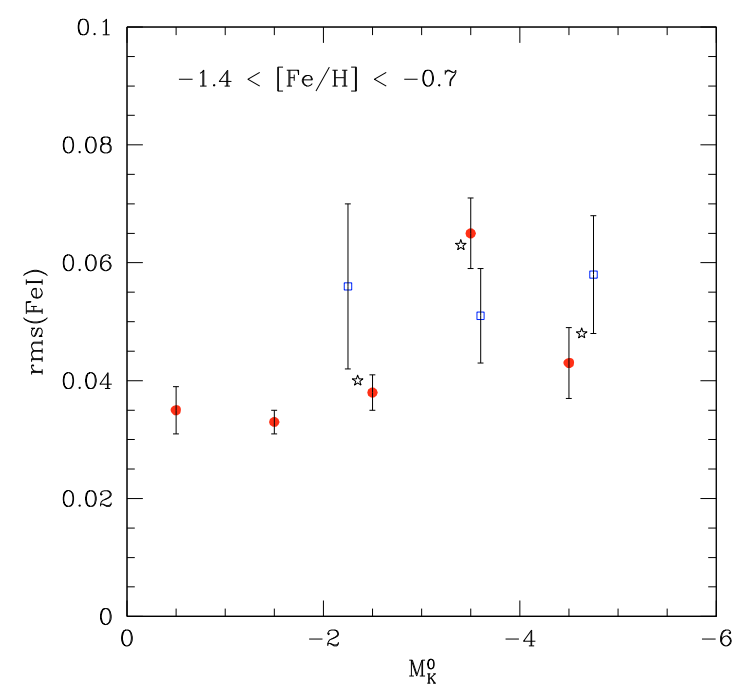

Fig. 6. Average differences between individual stars and the mean $[\mathrm{Fe} / \mathrm{H}]$ value in each cluster in the metallicity range $-1.4<[\mathrm{Fe} / \mathrm{H}]<$ -0.7 , as a function of the near IR de-reddened absolute magnitude $M_{K}^{0}$. Filled circles: stars observed with GIRAFFE; empty squares: stars observed with UVES. The open star symbols are the weighted average of residuals from UVES and GIRAFFE in the corresponding luminosity bin.

modulus and reddening in each cluster. We chose the $K$ band because (i) all near-IR magnitudes are from the 2MASS catalogue and they represent a very homogeneous dataset and (ii) this filter is much less sensitive to errors in the reddening estimate.

Finally, all stars were binned in luminosity intervals and the average residual and rms scatter from the mean was computed for each bin. These averages are listed in Table 3 and plotted in Fig. 6. We restricted this exercise to the metallicity range $[\mathrm{Fe} / \mathrm{H}]$ from -1.4 to -0.7 dex, where we have better quality data.

We can see that the brighter stars (for which the $S / N$ ratio is higher) show a larger scatter in the abundances of iron than the fainter stars. The difference is quite significant, considering the errors associated to the estimate of the scatter: in the bin $-2<M_{K}^{0}<-1$, with 271 stars, we obtain an rms scatter of $0.033 \pm 0.002$ from the GIRAFFE observations, whereas for the brighter bin $-4<M_{K}^{0}<-3$ (81 stars) we have $0.065 \pm 0.004$. This difference is statistically significant, at a level of more than $4 \sigma$. The results from the UVES spectra are essentially consistent with those derived from GIRAFFE (although with larger statistical errors owing to the much smaller number of stars available) and reveal a clear trend of the rms as a function of the luminosity.
This trend can be explained with the reasonable assumption that the atmosphere of each star (among the more luminous objects) has a distinct peculiarity or intrinsic time variability. Ita et al. (2002) found that very bright RGB stars in the LMC are indeed variables (likely pulsating). However, the stars considered here are considerably fainter, so that we are more inclined to attribute this variability to the large sizes (and small numbers) of convective cells with respect, e.g., to solar analogues 5 . As a consequence, there is an uncertainty (of the order of about 0.04-0.05 dex) in the Fe abundances derived from an instantaneous observation, since the convective elements of a red giant are so large that only a small number of them occupy the surface of the star at any time (Schwarzschild 1975; Gray et al. 2008). To account for this uncertainty we would need to assume that the temperature in the line formation region differs from star to stars, with an rms scatter of about $50 \mathrm{~K}$.

While Schwarzschild (1975) estimated as about 400 the number of convective cells in the atmosphere of a red giant, our sample is mostly formed by warmer stars. We then assume that the number of convective cells is larger, in our case, say about 1000 . Of those, only half are obviously visible and not all of them with the same weight, because of the limb darkening. Thus, we would get random fluctuations of about a factor 20 smaller than those expected at the star surface between the hotter regions (the top of the ascending columns) and the cooler regions (descending columns), having temperature differences by about $1000 \mathrm{~K}$. Hence, we could expect that random fluctuations of the temperature in the region of line formation in a giant are effectively of the order of $50 \mathrm{~K}$, even at similar effective temperatures. Following the same line of thought, one could expect that the luminosity is not perfectly constant, but should show fluctuations up to $0.01 \mathrm{mag}$ (over timescales of a few tens of days); the same would show up in fluctuations of radial velocity (over similar timescales and amplitude of about 200-300 m/s). These expectations seem to agree with observations of stars similar to those in our sample (the threshold being $-2<M_{V}<-1.4$, Cummings 1999; Carney et al. 2008).

In summary, since the spread measured from brighter stars could include the intrinsic noise due to the convection and pulsation (both in radial and non-radial mode), the implication is that it is better to use fainter stars in order to obtain the starto-star scatter. From these stars (practically restricted to those with GIRAFFE spectra) the typical rms in a cluster should be about 0.033 dex (see Table 3), or less, since this scatter value includes also the measurement errors.

Finally, we remark that the scatter seems to be larger for more metal-poor clusters. We speculate that this is in part due to possibly larger observational errors, but in part it could be due to an increased transparency of the atmosphere, hence pointing to a larger relevance of the $3 \mathrm{D}$-structure.

\subsection{Alternative scenario: contamination from $A G B$}

An alternative explanation of the larger scatter observed at higher luminosities (in particular in the magnitude bin $-4<$ $M_{K}<-3$ ) could be contamination from asymptotic giant branch (AGB) stars. Were this the case, we would expect that the sequences are sufficiently well separated (and the contamination

${ }^{5}$ Note, however, that this cannot be the full explanation. Studies of small amplitude variable red giants in LMC and SMC from the huge OGLE-II database (Soszynski et al. 2004) reveal that multi-periodic variability affects the majority of stars, with indications of non-radial oscillations for a sizable fraction of objects. 


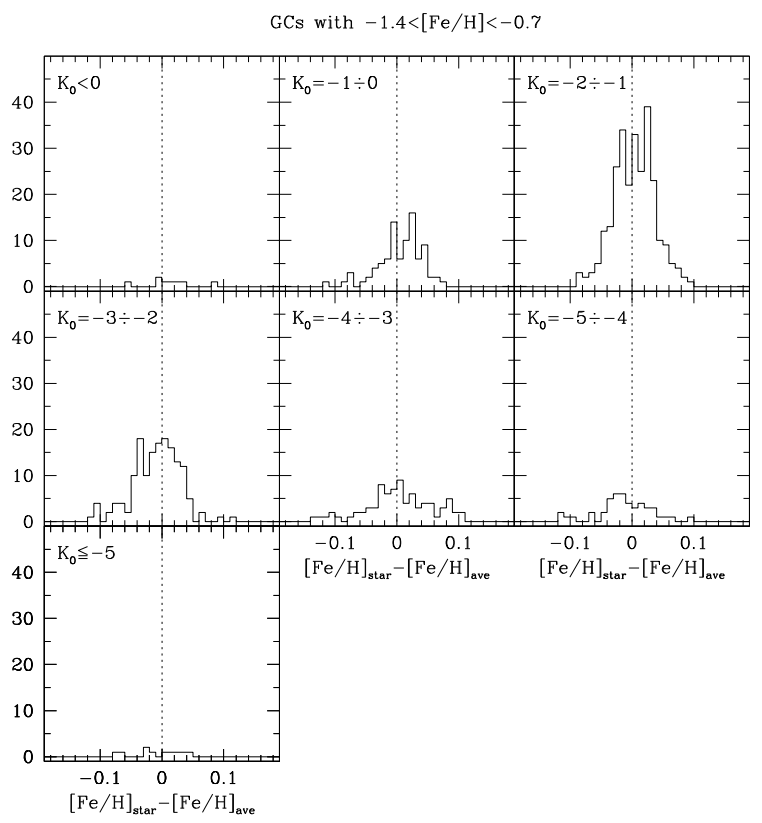

Fig. 7. Histograms of the residuals in $\mathrm{Fe}$ abundances in several magnitude bins for clusters in our sample with metallicity $-1.4<[\mathrm{Fe} / \mathrm{H}]<-0.7$.

negligible) at fainter magnitudes. On the other hand, the temperature difference between RGB and AGB is small, in the more luminous bin. However, the error would be larger where the two sequences are so close that cannot be distinguished, but separated enough to give a wrong temperature when the luminosity is used, as in the procedure adopted in our analysis (see Carretta et al. 2009a for further details).

In this scenario, we would expect a distribution essentially Gaussian in form at lower luminosities, where the contamination is negligible. At higher magnitudes, the distribution should become asymmetric, with a tail toward lower abundances given by the possible interlopers AGB stars for which the effective temperature (hence the abundance) would be underestimated. This asymmetry should be more pronounced in the bin $-4<M_{K}<-3$.

We checked this possible effect by computing the residuals in $\mathrm{Fe}$ abundances derived from GIRAFFE spectra in different bins of magnitudes, again restricting to the clusters in the metallicity range $-1.4<[\mathrm{Fe} / \mathrm{H}]<-0.7$ dex. The histogram of the residuals in various magnitude bins are plotted in Fig. 7

There seems to be no particular asymmetry in the range $-4<$ $M_{K}<-3$, where the distribution appears only slightly larger. In conclusion, this test seems to support the idea that the larger spread is due to the stellar atmospheres and to the variability provoked by the photospheric convection and stellar pulsation.

\section{A new homogeneous metallicity scale}

Having at hand an unprecedented large sample of GC red giants with homogeneously measured metal abundances, a logic step is to re-address the issue of an accurate metallicity scale for GCs. This is an indispensable tool in several issues, like age derivation (both absolute and relative to other clusters) of individual GCs as well as the study of age-metallicity relation for cluster populations.

In the following we provide the relations to transform previous metallicity scales against the one that can be defined on the basis of our UVES sample of stars.

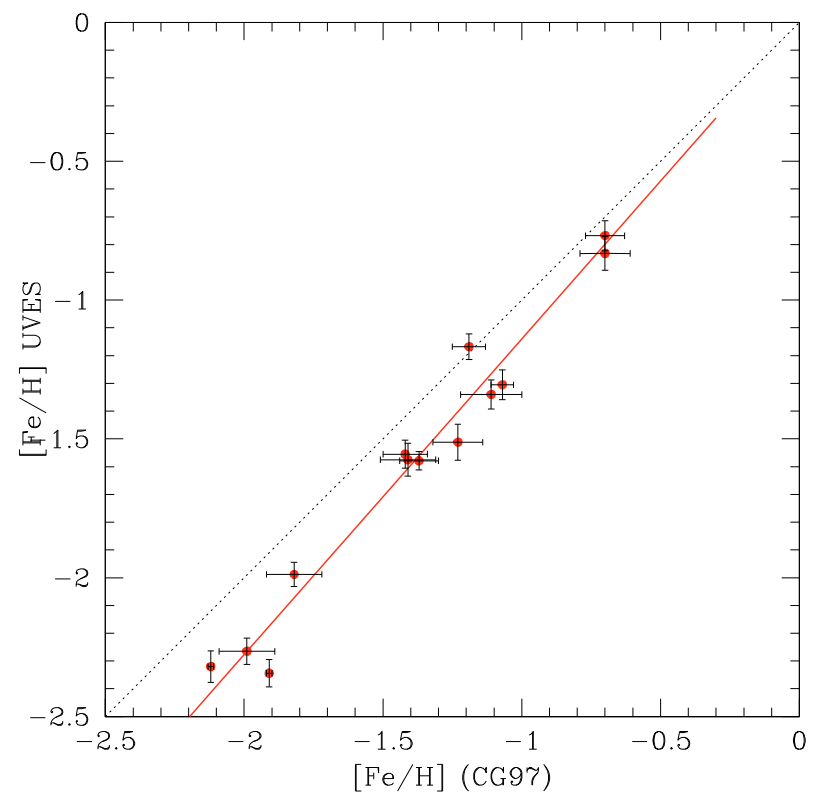

Fig. 8. Calibration of the CG97 metallicity scale vs average metal abundances derived by us from UVES spectra for 13 clusters in common. The dotted line is the equality line, the solid line is the linear regression obtained by least-squares fit to the points. Error bars are rms scatters of the mean.

Recalibration of the Carretta \& Gratton (1997; CG97) scale since its appearance, this metallicity scale has been widely used due to the effort made to use the most homogeneous approach. Briefly, the CG97 scale was derived from the re-analysis of a large sample $(\sim 160)$ of bright giants in $24 \mathrm{GCs}$, whose abundances were obtained from $E W \mathrm{~s}$ measured on high dispersion (at the time, $R \sim 30000$ ) CCD spectra, adjusted to a common system. All data were re-analysed using a common set of model atmospheres (the same we are using in the present study, from Kurucz 1993), a common set of high quality laboratory oscillator strengths $g f$ s for Fe I lines, and, whenever possible, the same colour-temperature scale (Cohen et al. 1978), based on the visual-near infrared colour $V-K$ available at the time. An improvement on that seminal work is that in the present analysis all the $E W \mathrm{~s}$ are measured with the same procedure, from higher resolution $(R \sim 43000)$ spectra all acquired with the same instrument (UVES). Moreover, atmospheric parameters $T_{\text {eff }}$ and $\log g$ are obtained for all stars using the same calibrations (on the Alonso et al. 1999 scale, based on the infrared flux-method) and the same accurate $K$ magnitudes from the recent 2MASS database.

We used 13 clusters in common between CG97 and the present analysis to provide a new calibration of the metallicity scale. The regression line is obtained by least-squares fit, by averaging values derived exchanging the independent and dependent variables, and is

$$
[\mathrm{Fe} / \mathrm{H}]_{\mathrm{UVES}}=1.137( \pm 0.060)[\mathrm{Fe} / \mathrm{H}]_{\mathrm{CG} 97}-0.003
$$

with $\sigma=0.094$ dex and correlation coefficient $r=0.98$ from 13 clusters. The comparison is shown in Fig. 8.

Our new $[\mathrm{Fe} / \mathrm{H}]$ values are systematically lower (by 0.19 dex on average, with $\sigma=0.11 \mathrm{dex}$ ) than those by CG97, and the difference increases with decreasing $[\mathrm{Fe} / \mathrm{H}]$. We must note, however, that the average values for the three most metal-poor clusters (NGC 4590, NGC 7078, and NGC 7099) are based in CG97 only on a few stars (from 2 to 4 ) in each cluster, compared to more than 10 stars per cluster in our new analysis. The above 
relation shows that there is almost only a multiplicative constant between the two scales and provides the transformation required to bring the CG97 scale onto the new system.

Part of the difference is certainly due to the adopted temperature scale. Since we observed different stars (farther away from the RGB tip in the present study) and different colours (2MASS rather than on the CIT system, Frogel et al. 1983) a very precise comparison is not possible. Anyway, the Alonso et al. scale is cooler: we found differences between 57 and $97 \mathrm{~K}$, for the same $V-K$ colours and different metallicities, with the larger difference found for more metal-poor GCs. Using the new Alonso et al. scale we would have had lower abundances also on the CG97 scale, by about 0.09-0.10 dex for the more metal-poor GCs and by only $0.02-0.03$ dex for the more metal-rich GCs, which accounts for most of the difference we found between the two scales. The metallicity found here for M 30 can be explained by the different temperatures: a star of magnitude similar to the two examined in CG97 has a temperature lower by about $140 \mathrm{~K}$ in our new analysis. This difference, larger than the average one for metal-poor GCs (about $100 \mathrm{~K}$ ), justifies the observed offset.

However, even including this correction we would still have an offset of about $0.08-0.12$ dex. Part of this residual difference is due to the $g f$ values: those currently adopted by us (see Gratton et al. 2003) are larger on average by $0.026 \pm 0.005$ dex. The remaining difference of $0.05-0.09$ dex are probably due to the $E W \mathrm{~s}$, measured on higher quality and higher resolution spectra and in a more homogeneous way in the present project.

Finally, we note that most of the scatter around the best fit is given (with opposite sign) by two clusters, NGC 6121 (M 4) and NGC 7099 (M 30). For the first case, the difference is essentially due to the anomalous value of the ratio $A_{V} / E(B-V)$, which was taken into account for this cluster in the present programme. The difference between the classical value 3.1 (CG97) and 4.0 (used in this work) implies a difference of $0.28 \mathrm{mag}$ in the correction of $V-K$, which in turn translates in a difference of about $200 \mathrm{~K}$ in the effective temperatures, just enough to shift M 4 on the best fit regression given by the other GCs.

Concerning NGC 7099 (M 30), the original data adopted by CG97 were from Minniti et al. (1993) who derived the effective temperatures from the spectra; these temperature were adopted also in CG97, due to the lack of $V-K$ colours for the two stars analysed.

Recalibration of the Zinn \& West (1984; ZW) scale - the other most used metallicity scale for globular clusters is the one defined by Zinn \& West (1984), based on a variety of integratedlight photometric and spectroscopic indices calibrated from the few echelle photographic spectra existing at the time. Its popularity was due to the ranking this scale provided for the first time for a large number of clusters; in fact, all 19 GCs of our project have a corresponding $[\mathrm{Fe} / \mathrm{H}]$ entry in the $\mathrm{ZW}$ scale.

The comparison between our average metallicities from UVES spectra and the $\mathrm{ZW}$ average $[\mathrm{Fe} / \mathrm{H}]$ values is shown in Fig. 9. A least-squares linear fit (again obtained by exchanging the independent and dependent variables and averaging the results) gives the following relation to transform $\mathrm{ZW}$ values to our new scale:

$$
[\mathrm{Fe} / \mathrm{H}]_{\mathrm{UVES}}=1.105( \pm 0.061)[\mathrm{Fe} / \mathrm{H}]_{\mathrm{ZW}}+0.160
$$

with $\sigma=0.143 \mathrm{dex}$ and correlation coefficient $r=0.97$ from 19 clusters.

On average, the two scales formally differ by only $0.01 \mathrm{dex}$ over the sampled metallicity range, but the scatter of the points is

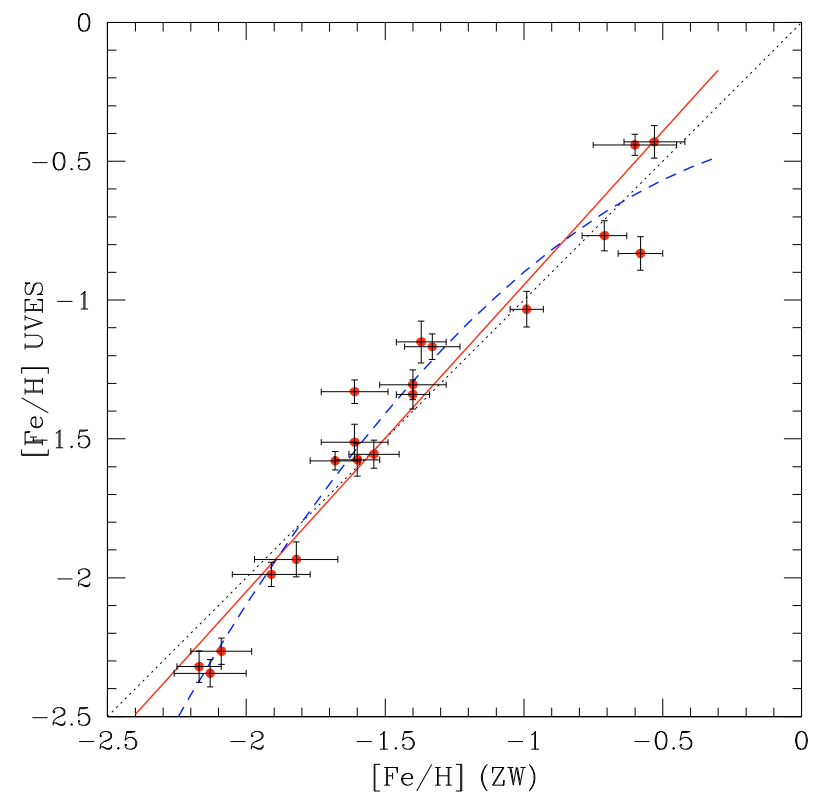

Fig. 9. Comparison of the $\mathrm{ZW}$ metallicity scale vs average $[\mathrm{Fe} / \mathrm{H}]$ values obtained by us from UVES spectra for all 19 clusters in our programme sample. The dotted line is the line of equality, the solid (red) line is the linear regression obtained by least-squares fit to the points and the dashed (blue) line is a second-order polynomial relation to the data. Error bars are rms scatters of the mean.

now clearly larger than in the case of the CG97 scale. By looking at Fig. 9 a better transformation is obtained when a second-order polynomial is used:

$$
\begin{aligned}
{[\mathrm{Fe} / \mathrm{H}]_{\text {UVES }}=} & -0.413( \pm 0.027)+0.130( \pm 0.289)[\mathrm{Fe} / \mathrm{H}]_{\mathrm{ZW}} \\
& -0.356( \pm 0.108)[\mathrm{Fe} / \mathrm{H}]_{\mathrm{ZW}}{ }^{2}
\end{aligned}
$$

with $\sigma=0.119$ dex and correlation coefficient $r=0.98$ from 19 clusters. We made an analysis of variance and tested with an F-test the statistical significance of the regression with multiple components and the significance of the coefficient of the highest degree. Both result highly significant, and the resulting scatter in the transformation is decreased by using the second order equation above.

We note however that metallicities on the ZW scale are obtained by averaging the results from a heterogeneous series of indices, calibrated on the best metal abundances from high dispersion spectroscopy available at the time (Cohen 1983; Frogel et al. 1983). A better approach is to directly calibrate the original and homogeneous $Q_{39}$ index by Zinn \& West (1984), available for a large sample of GCs, on the new metallicities presented here and use it in addition to other homogeneous estimate of $[\mathrm{Fe} / \mathrm{H}]$ to obtain an accurate ranking in metal abundance for Galactic GCs. This is done in the Appendix.

Recalibration of the Kraft \& Ivans (2003; KI03) scale - in Fig. 10 we show the comparison of our $[\mathrm{Fe} / \mathrm{H}]$ values from UVES with those of the metallicity scale by Kraft \& Ivans (2003), not much used in spite of being based on the homogeneous re-analysis of $E W \mathrm{~s}$ of Fe II lines from high resolution spectra. We adopted their values from the Kurucz model with convective overshooting turned on (their Table 4, Col. 6) for consistency with the models used in the present work.

The relation to transform the KI03 values on our new scale is:

$[\mathrm{Fe} / \mathrm{H}]_{\mathrm{UVES}}=0.917( \pm 0.038)[\mathrm{Fe} / \mathrm{H}]_{\mathrm{KI}}-0.181$ 


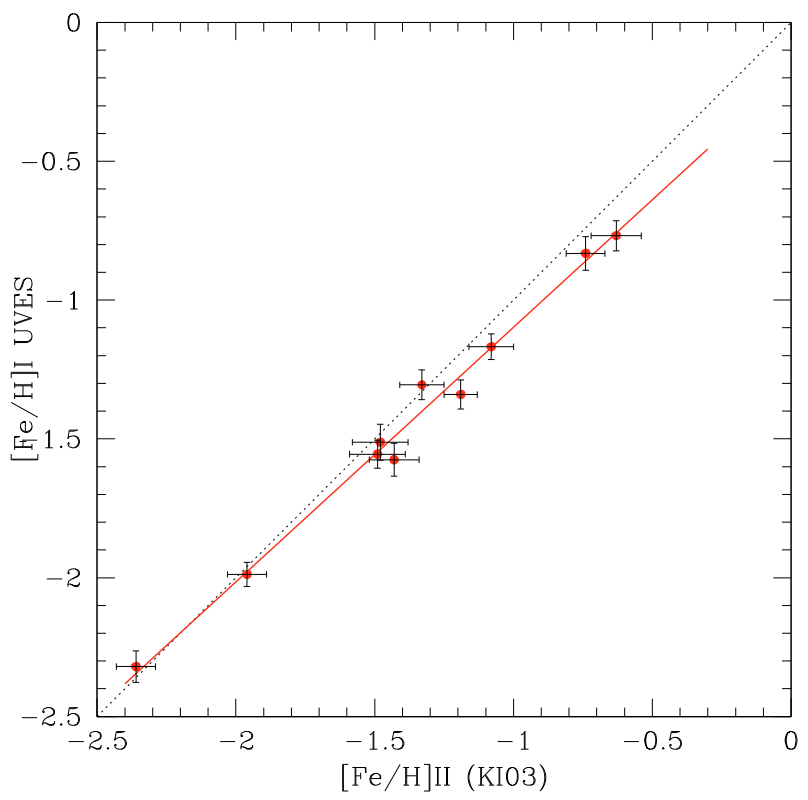

Fig. 10. Comparison of the Kraft \& Ivans (2003) metallicity scale based on $\mathrm{Fe}$ II abundances vs average $[\mathrm{Fe} / \mathrm{H}]$ values obtained by us from $\mathrm{Fe}$ I lines measured on UVES spectra for 10 clusters in common. The dotted line is the identity line, the solid (red) line is the linear regression obtained by least-squares fit to the points. Error bars are rms scatters of the mean.

with a scatter of only $\sigma=0.056$ dex and correlation coefficient $r=0.99$ from 10 clusters. Surprisingly, the agreement we found is quite good, on average the two scale differ only by 0.07 dex (our averages being lower) but this difference is scarcely significant (the scatter about the mean being 0.07 dex as well). The difference increases with increasing metallicity, and is on average null at low $[\mathrm{Fe} / \mathrm{H}]$ values.

This finding is rather strange because the basic reason for which Kraft and Ivans used Fe II is that it is generally assumed that currently available one-dimensional LTE model atmospheres give a correct representation of abundances from singly ionized $\mathrm{Fe}$ lines, but not of the abundances from Fe I lines, more affected by departures from the LTE assumption. Hence, they concluded that the safest approach was to rely on Fe II lines alone. However, Fig. 10 seems to question these conclusion for two related reasons. First, because of the good agreement we found on average, being our abundances derived from a neutral specie and theirs from a singly ionized specie; second, because departures from LTE are expected to be larger where the stellar atmospheres become more transparent and less line-blanketed, i.e. the effects increase toward lower metallicities, whereas just the opposite is shown by the comparison. At the moment, we have no clear explanation for this occurrence; we only note that our samples are composed by stars not so close to the RGB tip, where NLTE effects might be more relevant.

Recalibration of the near-infrared Ca II scale (Rutledege et al 1997; R97) - finally, we provide here a new calibration of the metallicity scale based on the strength of the near-infrared Ca II triplet, used in particular to estimate the metallicity of stars in distant systems, such as the nearby dwarf spheroidals, because the triplet is measurable even at large distances.

In Fig. 11 we show the comparison between our UVES metallicities and the values of the reduced strength $W^{\prime}(\mathrm{Ca}$ II) of the near-infrared Ca triplet taken from Rutledge et al. (1997;

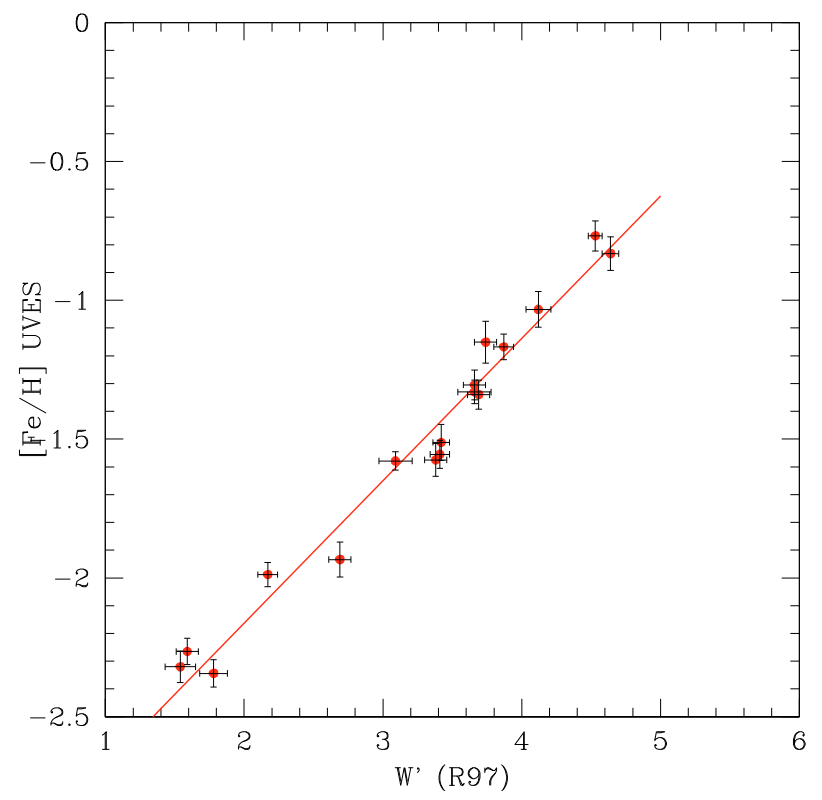

Fig. 11. Comparison of our $[\mathrm{Fe} / \mathrm{H}]$ average values with the reduced strength of the near-infrared Ca II triplet $W^{\prime}$ from Rutledge et al. (1997) for 17 clusters in common. The superimposed solid (red) line is the linear regression obtained by least-squares fit to the points. Error bars are rms scatters of the mean.

R97). The two variables are linearly correlated over the whole range defined by the clusters analysed in the present paper $\left(-2.4 \lesssim[\mathrm{Fe} / \mathrm{H}] \lesssim-0.8\right.$; unfortunately, values of $W^{\prime}$ are not given for NGC 6388 or NGC 6441). The transformation of the reduced strength $W^{\prime}$ to the UVES metallicities

$[\mathrm{Fe} / \mathrm{H}]_{\mathrm{UVES}}=0.513( \pm 0.022) W_{R 97}^{\prime}-3.189$

(with a correlation coefficient $r=0.99$ and a rms scatter of only $\sigma=0.086$ dex from 17 clusters) is therefore strictly only applicable up to the metallicity of NGC 104 (47 Tuc), [Fe/H] = -0.77 dex. However, from the abundance analysis of two very metal rich bulge clusters, Carretta et al. (2001) already noted that the index $W^{\prime}$ might deviate from linearity at high metallicity, whereas in their work Rutledge et al. (1997) claimed that a linear relation existed between $W^{\prime}$ and $[\mathrm{Fe} / \mathrm{H}]_{\mathrm{CG} 97}$, simply because results for metal-rich clusters were not available at the time. With the present sample we cannot extend the calibration further, but in the Appendix we add two near solar metallicity bulge clusters to obtain a more extended calibration of this index, to be inserted in a final compilation of GC metallicities.

\section{Summary}

Within our survey of 19 Galactic GCs with FLAMES (UVES and GIRAFFE), we have measured the iron abundance of about 2000 RGB stars in a accurate and homogeneous way (determination of atmospheric parameters, measure of $E W \mathrm{~s}$, spectroscopic analysis, etc). In the present paper we exploit this huge, homogeneous data set, covering (almost) the entire range of GC metallicity in our Galaxy and a summary of our findings follows:

i) We obtain a measure of the intrinsic spread of iron in GCs from the rms scatter of our metallicities. We confirm that the cosmic scatter in Fe of most GCs is very small: the upper limit to this scatter is less than 0.05 dex, i.e., the iron abundance is homogeneous within $12 \%$ in each GC, on average. 
ii) The rms scatter we find for the UVES sample within each GC is larger than the one derived for the GIRAFFE sample. This can be ascribed to the different magnitude ranges observed. The UVES stars are usually brighter, nearer the RGB tip, and may suffer from larger inadequacies in the treatment of atmospheres and intrinsic stellar variability.

iii) This is confirmed by a increase in scatter for brighter stars also in the GIRAFFE samples, that is not explained by AGB contamination, and that is larger for metal-poor GCs.

iv) There are interesting correlations between the rms scatter in Fe and several GC parameters, like $M_{V}, T_{\text {eff }}^{\max }(\mathrm{HB}), \alpha-$ element abundance, that seem to indicate a better capability of more massive clusters to self-enrich.

v) We recalibrate several metallicity scales of GCs using our 19 precise, homogeneous values; we have done it for the GC97, ZW, KI03, and R97 ones, giving transformation equations.

vi) Finally, in the Appendix we give $[\mathrm{Fe} / \mathrm{H}]$ on our new scale for all clusters in the Harris (1996) compilation.

Acknowledgements. We wish to thank the ESO Service Mode personnel for their efforts. We also thank the referee for his/her useful comments. This publication makes use of data products from the Two Micron All Sky Survey, which is a joint project of the University of Massachusetts and the Infrared Processing and Analysis Center/California Institute of Technology, funded by the National Aeronautics and Space Administration and the National Science Foundation. This work was partially funded by the Italian MIUR under PRIN 2003029437. We also acknowledge partial support from the grant INAF 2005 "Experimental nucleosynthesis in clean environments". S.L. is grateful to the DFG cluster of excellence "Origin and Structure of the Universe" for support.

\section{Appendix A: Cluster metallicities on a new homogeneous UVES scale}

In this Section, we provide an updated compilation of cluster metallicity for all the Galactic GCs included in the Harris (1996) catalogue referred to modern measurements of stellar abundances from high dispersion spectroscopy. We use as reference "pillars" the accurate and homogeneous $[\mathrm{Fe} / \mathrm{H}]$ values derived in our project from high resolution UVES spectra for 214 giants stars in 19 GCs. The very good agreement between these abundances and those obtained for stars observed also with GIRAFFE means that our scale is supported by the observed metallicities of almost 2000 red giants, all analysed in the same way.

In our approach we selected two sets of data to be included in our new calibration:

(i) recent and homogeneous metallicities from high resolution spectroscopy of stars in a limited number of GCs (CG97, 24 clusters; Kraft \& Ivans 2003, 16 clusters)

(ii) metallicity dependent indices homogeneously derived for large; samples of GCs ( $Q_{39}$ from Zinn \& West 1984, 60 clusters; $W^{\prime}$ from Rutledge et al. 1997, 69 clusters).

Our metallicities are valid only over the metallicity range sampled by our programme clusters. In the very low metallicity end this is not an issue, since we analysed some of the most metal-poor clusters (NGC 7078, NGC 7099, NGC 4590, and NGC 6397). However, problems might arise at the high metallicity extreme, near solar $[\mathrm{Fe} / \mathrm{H}]$ values. Since the most metal-rich clusters are NGC 6388 and NGC $6441([\mathrm{Fe} / \mathrm{H}]$ about $-0.4 \mathrm{dex})$, metallicities beyond this limit would be derived in extrapolation, and this is a risky procedure.

To alleviate this problem, we add to our 19 GCs two very metal-rich clusters, whose metal abundances were

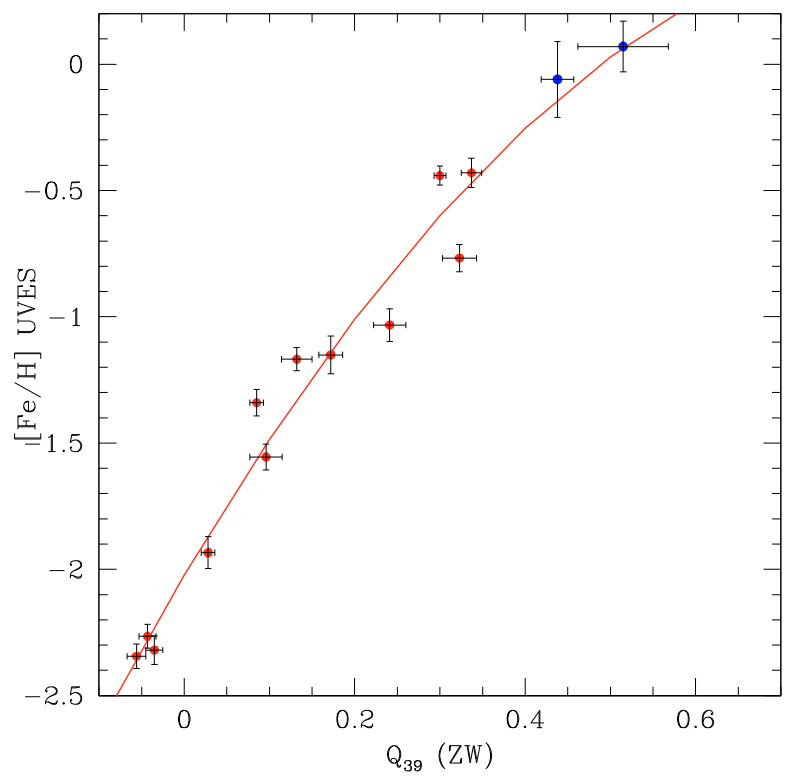

Fig. A.1. Calibration of the $Q_{39}$ index from Zinn \& West (1984) using our $[\mathrm{Fe} / \mathrm{H}]$ values from high resolution spectroscopy. Filled red circles are our clusters with UVES spectra; blue filled squares are the two metal-rich bulge clusters whose metallicities were analysed (or revised) in Carretta et al. (2001), based on Keck HIRES spectra.

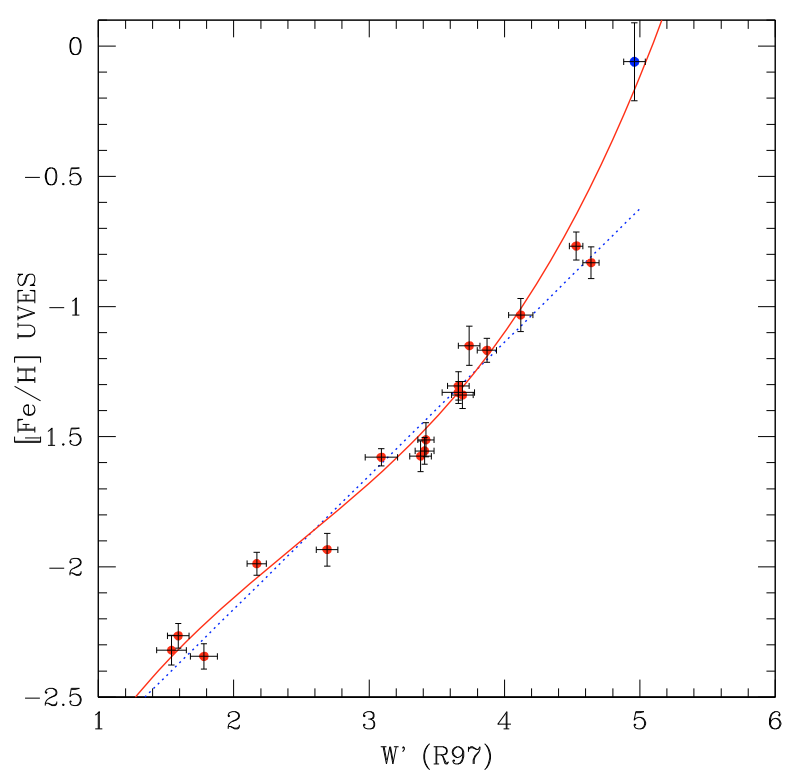

Fig. A.2. Calibration of the reduced strength $W^{\prime}$ of the calcium triplet from Rutledge et al. (1997) after adding the metal-rich bulge cluster NGC 6553 (NGC 6528 does not have $W^{\prime}$ measured by Rutledge et al.) from Carretta et al. (2001; blue filled square). The blue dotted line is the linear regression of Fig. 11 and the red solid line the cubic spline fit to the data.

newly analysed (or revised) in Carretta et al. (2001): NGC $6528([\mathrm{Fe} / \mathrm{H}]=+0.07 \mathrm{dex}, \sigma=0.1 \mathrm{dex})$ and NGC 6553 $([\mathrm{Fe} / \mathrm{H}]=-0.06 \mathrm{dex}, \sigma=0.15 \mathrm{dex})$. For these two clusters the analysis is of course not strictly homogeneous with the other $19 \mathrm{GCs}$, however the $g f$ scale is very similar and their addition to the sample is unavoidable to obtain a reasonable calibration of the metal rich end of the GC scale, as we will see below.

In Fig. A. 1 we show the calibration of the $Q_{39}$ index from Zinn \& West (1984) using the 12 GCs with UVES metallicities 
Table A.1. Compilation of cluster metallicities on the present scale.

\begin{tabular}{|c|c|c|c|c|c|c|c|c|c|}
\hline$\overline{\mathrm{GC}}$ & Other & {$[\mathrm{Fe} / \mathrm{H}]$} & err & Note & $\overline{\mathrm{GC}}$ & Other & {$[\mathrm{Fe} / \mathrm{H}]$} & err & Note \\
\hline NGC 104 & 47Tuc & -0.76 & 0.02 & 1 & Ter2 & & -0.29 & 0.09 & 2 \\
\hline NGC 288 & & -1.32 & 0.02 & 1 & NGC 6366 & & -0.59 & 0.08 & 1 \\
\hline NGC 362 & & -1.30 & 0.04 & 1 & NGC 6362 & & -1.07 & 0.05 & 1 \\
\hline NGC 1261 & & -1.27 & 0.08 & 1 & Ter4 & & -1.62 & 0.09 & 2 \\
\hline Pall & & -0.51 & 0.09 & 2 & HP1 & & -1.57 & 0.09 & 2 \\
\hline AM1 & & -1.84 & 0.09 & 2 & LILLER1 & & +0.40 & 0.09 & 2 \\
\hline Eridanus & & -1.44 & 0.08 & 1 & NGC 6380 & TON1 & -0.40 & 0.09 & 2 \\
\hline $\mathrm{Pa} 2$ & & -1.29 & 0.09 & 2 & Ter1 & & -1.29 & 0.09 & 2 \\
\hline NGC 1851 & & -1.18 & 0.08 & 1 & NGC 6388 & & -0.45 & 0.04 & 1 \\
\hline NGC 1904 & M 79 & -1.58 & 0.02 & 1 & NGC 6402 & M 14 & -1.39 & 0.09 & 2 \\
\hline NGC 2298 & & -1.96 & 0.04 & 1 & NGC 6401 & & -1.01 & 0.14 & 1 \\
\hline NGC 2419 & & -2.20 & 0.09 & 2 & NGC 6397 & & -1.99 & 0.02 & 1 \\
\hline NGC 2808 & & -1.18 & 0.04 & 1 & Pal6 & & -1.06 & 0.09 & 2 \\
\hline E3 & & -0.73 & 0.09 & 2 & NGC 6424 & & -2.36 & 0.09 & 2 \\
\hline $\mathrm{Pal} 3$ & & -1.67 & 0.08 & 1 & Ter5 & & +0.16 & 0.09 & 2 \\
\hline NGC 3201 & & -1.51 & 0.02 & 1 & NGC 6440 & & -0.20 & 0.14 & 1 \\
\hline Pal4 & & -1.46 & 0.08 & 1 & NGC 6441 & & -0.44 & 0.07 & 1 \\
\hline NGC 4147 & & -1.78 & 0.08 & 1 & NGC 6453 & & -1.48 & 0.14 & 1 \\
\hline NGC 4372 & & -2.19 & 0.08 & 1 & Ter6 & & -0.40 & 0.09 & 2 \\
\hline Rup106 & & -1.78 & 0.08 & 1 & UKS1 & & -0.40 & 0.09 & 2 \\
\hline NGC 4590 & M 68 & -2.27 & 0.04 & 1 & NGC 6496 & & -0.46 & 0.07 & 1 \\
\hline NGC 4833 & & -1.89 & 0.05 & 1 & Ter9 & & -2.07 & 0.09 & 2 \\
\hline NGC 5024 & M 53 & -2.06 & 0.09 & 2 & NGC 6517 & & -1.24 & 0.14 & 1 \\
\hline NGC 5053 & & -2.30 & 0.08 & 1 & NGC 6522 & & -1.45 & 0.08 & 1 \\
\hline NGC 5139 & OCEN & -1.64 & 0.09 & 2 & NGC 6535 & & -1.79 & 0.07 & 1 \\
\hline NGC 5272 & M 3 & -1.50 & 0.05 & 1 & NGC 6528 & & +0.07 & 0.08 & 1 \\
\hline NGC 5286 & & -1.70 & 0.07 & 1 & NGC 6539 & & -0.53 & 0.14 & 1 \\
\hline AM4 & & -2.07 & 0.09 & 2 & NGC 6544 & & -1.47 & 0.07 & 1 \\
\hline NGC 5466 & & -2.31 & 0.09 & 2 & NGC 6541 & & -1.82 & 0.08 & 1 \\
\hline NGC 5634 & & -1.93 & 0.09 & 2 & NGC 6553 & & -0.16 & 0.06 & 1 \\
\hline NGC 5694 & & -2.02 & 0.07 & 1 & NGC 6558 & & -1.37 & 0.14 & 1 \\
\hline IC 4499 & & -1.62 & 0.09 & 2 & IC1276 & Pal7 & -0.65 & 0.09 & 2 \\
\hline NGC 5824 & & -1.94 & 0.14 & 1 & NGC 6569 & & -0.72 & 0.14 & 1 \\
\hline Pal5 & & -1.41 & 0.09 & 2 & NGC 6584 & & -1.50 & 0.09 & 2 \\
\hline NGC 5897 & & -1.90 & 0.06 & 1 & NGC 6624 & & -0.42 & 0.07 & 1 \\
\hline NGC 5904 & M 5 & -1.33 & 0.02 & 1 & NGC 6626 & & -1.46 & 0.09 & 2 \\
\hline NGC 5927 & & -0.29 & 0.07 & 1 & NGC 6638 & & -0.99 & 0.07 & 1 \\
\hline NGC 5946 & & -1.29 & 0.14 & 1 & NGC 6637 & M69 & -0.59 & 0.07 & 1 \\
\hline NGC 5986 & & -1.63 & 0.08 & 1 & NGC 6642 & & -1.19 & 0.14 & 1 \\
\hline Pal14 & & -1.63 & 0.08 & 1 & NGC 6652 & & -0.76 & 0.14 & 1 \\
\hline NGC 6093 & M 80 & -1.75 & 0.08 & 1 & NGC 6656 & M 22 & -1.70 & 0.08 & 1 \\
\hline NGC 6101 & & -1.98 & 0.07 & 1 & Pal8 & & -0.37 & 0.14 & 1 \\
\hline NGC 6121 & M4 & -1.18 & 0.02 & 1 & NGC 6681 & M 70 & -1.62 & 0.08 & 1 \\
\hline NGC 6144 & & -1.82 & 0.05 & 1 & NGC 6712 & & -1.02 & 0.07 & 1 \\
\hline NGC 6139 & & -1.71 & 0.09 & 2 & NGC 6715 & M 54 & -1.44 & 0.07 & 1 \\
\hline NGC 6171 & M 107 & -1.03 & 0.02 & 1 & NGC 6717 & Pal9 & -1.26 & 0.07 & 1 \\
\hline NGC 6205 & M 13 & -1.58 & 0.04 & 1 & NGC 6723 & & -1.10 & 0.07 & 1 \\
\hline NGC 6218 & M 12 & -1.33 & 0.02 & 1 & NGC 6749 & & -1.62 & 0.09 & 2 \\
\hline NGC 6229 & & -1.43 & 0.09 & 2 & NGC 6752 & & -1.55 & 0.01 & 1 \\
\hline NGC 6235 & & -1.38 & 0.07 & 1 & NGC 6760 & & -0.40 & 0.14 & 1 \\
\hline NGC 6254 & M 10 & -1.57 & 0.02 & 1 & NGC 6779 & M 56 & -2.00 & 0.09 & 2 \\
\hline NGC 6256 & & -0.62 & 0.09 & 2 & ARP2 & & -1.74 & 0.08 & 1 \\
\hline Pal15 & & -2.10 & 0.08 & 1 & NGC 6809 & M55 & -1.93 & 0.02 & 1 \\
\hline NGC 6266 & M 62 & -1.18 & 0.07 & 1 & Pal11 & & -0.45 & 0.08 & 1 \\
\hline NGC 6273 & M 19 & -1.76 & 0.07 & 1 & NGC 6838 & M 71 & -0.82 & 0.02 & 1 \\
\hline NGC 6284 & & -1.31 & 0.09 & 2 & NGC 6864 & M 75 & -1.29 & 0.14 & 1 \\
\hline NGC 6287 & & -2.12 & 0.09 & 2 & NGC 6934 & & -1.56 & 0.09 & 2 \\
\hline NGC 6293 & & -2.01 & 0.14 & 1 & NGC 6981 & M 72 & -1.48 & 0.07 & 1 \\
\hline NGC 6304 & & -0.37 & 0.07 & 1 & NGC 7006 & & -1.46 & 0.06 & 1 \\
\hline NGC 6316 & & -0.36 & 0.14 & 1 & NGC 7078 & M 15 & -2.33 & 0.02 & 1 \\
\hline NGC 6325 & & -1.37 & 0.14 & 1 & NGC 7089 & M2 & -1.66 & 0.07 & 1 \\
\hline NGC 6341 & M92 & -2.35 & 0.05 & 1 & NGC 7099 & M 30 & -2.33 & 0.02 & 1 \\
\hline NGC 6333 & M9 & -1.79 & 0.09 & 2 & Pal12 & & -0.81 & 0.08 & 1 \\
\hline NGC 6342 & & -0.49 & 0.14 & 1 & Pal13 & & -1.78 & 0.09 & 2 \\
\hline NGC 6356 & & -0.35 & 0.14 & 1 & NGC 7492 & & -1.69 & 0.08 & 1 \\
\hline NGC 6355 & & -1.33 & 0.14 & 1 & Ter7 & & -0.12 & 0.08 & 1 \\
\hline NGC 6352 & & -0.62 & 0.05 & 1 & & & & & \\
\hline
\end{tabular}

$[\mathrm{Fe} / \mathrm{H}]$ values are the weighted average of metallicities from the present work (UVES values), GC97, KI03, and the recalibration of the Q39 and $W^{\prime}$ indices. $2[\mathrm{Fe} / \mathrm{H}]$ values are those from the Harris (1996) updated catalogue with an offset of 0.025 dex added.

in common with that work and the two added clusters NGC 6528 and NGC 6553. From this figure it is clearly evident that the run of $Q_{39}$ as a function of metal abundance is not linear in the high metallicity regime. The fitting second-order polynomial is

$$
\begin{aligned}
{[\mathrm{Fe} / \mathrm{H}]_{\mathrm{UVES}}=} & -2.023( \pm 0.038)+5.699( \pm 0.589) Q_{39} \\
& -3.188( \pm 1.320) Q_{39}^{2}
\end{aligned}
$$

with $\sigma=0.14$ dex and correlation coefficient $r=0.99$ from $14 \mathrm{GCs}$.
Although anchored to only one cluster, NGC 6553, in the near solar metallicity region, the new calibration of the calcium triplet reduced strength, of the form

$$
\begin{aligned}
{[\mathrm{Fe} / \mathrm{H}]_{\mathrm{UVES}}=} & -3.632( \pm 0.026)+1.233( \pm 1.014) W^{\prime} \\
& -0.326( \pm 0.329) W^{\prime 2}+0.044( \pm 0.033) W^{\prime 3}
\end{aligned}
$$

(with $\sigma=0.11$ dex and a correlation coefficient $r=0.99$ from 18 GCS) is a better fit (see Fig. A.2) than the simple linear 
regression previously shown (and indicated by the dotted line in this figure).

Moreover, from Figs. A.1 and A.2 we note that simple linear extrapolations would result in $[\mathrm{Fe} / \mathrm{H}]$ values for clusters with $[\mathrm{Fe} / \mathrm{H}]>-0.5$ that would be overestimated from the $Q_{39}$ index and underestimated when using the $W^{\prime}$ index. This fact underlines the risks of a too crude extrapolation procedure, and we believe that this justifies the inclusion of NGC 6553 and NGC 6528 among our calibrating clusters.

We used these transformations to derive $[\mathrm{Fe} / \mathrm{H}]$ values on our scale and the previous relations of Sect. 5 to transform metallicities from Carretta \& Gratton (1997) and from Kraft \& Ivans (2003) to the present scale. Finally, we computed the weighted average of the metallicity for each cluster, assuming as weights the rms scatters of the fitting relations in each case. For our UVES based metal abundances we adopted internal errors (see Carretta et al. 2009b) as weighting factor.

The resulting metallicities are listed in Table A.1 for 95 GCs in the Harris (1996) catalogue for which this average was possible. Associated errors are $1 \sigma \mathrm{rms}$ of the weighted average and they represent the errors associated with the metallicity with respect to the metallicity scale/ranking defined in this paper.

The other 38 entries in the Harris catalogue have no determinations of $[\mathrm{Fe} / \mathrm{H}]$ from homogeneous high resolution spectroscopy or compilation of metallicity-dependent indices. In general, these are distant clusters, not included in any of the previous main studies. Their metal abundance in the Harris catalogue is based on a variety of metallicity indicators. For these objects we proceeded as follows. The comparison of our weighted average $[\mathrm{Fe} / \mathrm{H}]$ values with metallicity entries in the Harris catalogue for 94 clusters in common ${ }^{6}$ provides a slope equal to unity (when metallicity from Harris is used as independent variable) with an offset of 0.025 dex. Hence, for the 38 remaining clusters we simply added this offset to the $[\mathrm{Fe} / \mathrm{H}]$ values listed in the Harris catalogue (flag 2 in Table A.1). In this case, the associated error is simply the rms scatter from the comparison of the 94 clusters, i.e. 0.09 dex.

\section{References}

Alonso, A., Arribas, S., \& Martinez-Roger, C. 1999, A\&AS, 140, 261 Alonso, A., Arribas, S., \& Martinez-Roger, C. 2001, A\&A, 376, 1039 Bedin, L., Piotto, G., Anderson, J., et al. 2004, ApJ, 605, L125

Bellazzini, M., Ibata, R. A., Chapman, S. C., et al. 2008, AJ, 136, 1147 Bragaglia, A., Carretta, E., Gratton, R. G., et al. 2001, AJ, 121, 327

Carney, B. W., Latham, D. W., Stefanik, R. P., \& Laird, J. B. 2008, AJ, 135, 196 Carretta, E., \& Gratton, R. G. 1997, A\&AS, 121, 95

Carretta, E., Cohen, J. G., Gratton, R. G., \& Behr, B. B. 2001, AJ, 122, 1469

Carretta, E., Gratton, R. G., Bragaglia, A., Bonifacio, P., \& Pasquini, L. 2004, A\&A, 416, 925

Carretta, E., Bragaglia, A., Gratton, R. G., et al. 2006, A\&A, 450, 523 (Paper I)

Carretta, E., Bragaglia, A., Gratton, R. G., Lucatello, S., \& Momany, Y. 2007a, A\&A, 464, 927 (Paper II)

Carretta, E., Bragaglia, A., Gratton, R. G., et al. 2007b, A\&A, 464, 939 (Paper IV)
Carretta, E., Bragaglia, A., Gratton, R. G., et al. 2007c, A\&A, 464, 967 (Paper VI)

Carretta, E., Bragaglia, A., Gratton, R. G., et al. 2009a, A\&A, 505, 117 (Paper VII)

Carretta, E., Bragaglia, A., Gratton, R. G., \& Lucatello, S. 2009b, A\&A, 505, 139 (Paper VIII)

Carretta, E., Bragaglia, A., Gratton, R. G., et al. 2009c, A\&A, submitted Casetti-Dinescu, D. I., Girard, T. M., Herrera, D., et al. 2007, AJ, 134, 195

Charbonnel, C. 1994, A\&A, 282, 811

Charbonnel, C. 1995, ApJ, 453, L41

Charbonnel, C., \& Zahn, J.-P. 2007, A\&A, 467, L15

Cohen, J. G. 1983, ApJ, 270, 654

Cohen, J. G., Frogel, J. A., \& Persson, S. E. 1978, ApJ, 222, 165

Cummings, I. N. 1999, J Astron. Data, 5, 2

De Angeli, F., et al. 2005, AJ, 122, 3171

Decressin, T., Baumgardt, H., Kroupa, P., Meynet, G., \& Charbonnel, C. 2009, IAU Symp. 258, ed. D. Soderblom et al., 265

D’Ercole, A., Vesperini, E., D’Antona, F., McMillan, S. L. W., \& Recchi, S. 2008, MNRAS, 391, 825

Denisenkov, P. A., \& Denisenkova, S. N. 1989, A.Tsir., 1538, 11

Dinescu, D. I., Girard, T. M., \& van Altena, W. F. 1999, AJ, 117, 1792

Djorgovski, S., Piotto, G., \& Capaccioli, M. 1993, AJ, 105, 2148

Eggleton, P. P., Dearborn, D. S. P., \& Lattanzio, J. C. 2007, IAUS, 239, 286

Frogel, J. A., Cohen, J. G., \& Persson, S. E. 1983, ApJ, 275, 773

Gnedin, O. Y., Zhao, H. S., Pringle, J. E., et al. 2002, ApJ, 568, L23

Gratton, R. G. 1988, Rome Obs. Preprint Ser., 29

Gratton, R. G., Bonifacio, P., Bragaglia, A., et al. 2001, A\&A, 369, 87

Gratton, R. G., Carretta, E., Claudi, R., Lucatello, S., \& Barbieri, M. 2003, A\&A, 404, 187

Gratton, R. G., Sneden, C., \& Carretta, E. 2004, ARA\&A, 42, 385

Gratton, R. G., Lucatello, S., Bragaglia, A., et al. 2006, A\&A, 455, 271 (Paper III)

Gratton, R. G., Lucatello, S., Bragaglia, A., et al. 2007, A\&A, 464, 953 (Paper V)

Gratton, R. G., Carretta, E., Bragaglia, A., Lucatello, S., \& D’Orazi, V. 2009, A\&A, submitted

Gray, D. F., Carney, B. W., \& Yong, D. 2008, AJ, 135, 2033

Harris, W. E. 1996, AJ, 112, 1487

Ita, Y., Tanabé, T., Matsunaga, N., et al. 2002, MNRAS, 337, L31

Kraft, R. P., \& Ivans, I. I. 2003, PASP, 115, 143

Kurucz, R. L. 1993, CD-ROM 13, Smithsonian Astrophysical Observatory, Cambridge

Langer, G. E., Hoffman, R., \& Sneden, C. 1993, PASP, 105, 301

Larson, R. B. 1987, in Nearby normal galaxies, ed. S. Faber (NY: Springer), 26

Marino, A. F., Milone, A., Piotto, G., et al. 2009, A\&A, 505, 1099

Melendez, J., \& Cohen, J. G. 2009, ApJ, 699, 2017

Minniti, D., Geisler, D., Peterson, R. C., \& Claria, J. J. 1993, ApJ, 413, 548

Piotto, G., Villanova, S., Bedin, L. R., et al. 2005, ApJ, 621, 777

Prantzos, N., \& Charbonnel, C. 2006, A\&A, 458, 135

Ramirez, S. V., \& Cohen, J. G. 2002, AJ, 123, 3277

Recio Blanco, A., Aparicio, A., Piotto, G., De Angeli, F., \& Djorgovski, S. G. 2006, A\&A, 452, 875

Rosenberg, A., Saviane, I., Piotto, G., \& Aparicio, A. 1999, AJ, 118, 2306

Rutledge, G. A., Hesser, J. E., \& Stetson, P. B. 1997, PASP, 109, 907

Schwarzschild, M. 1975, ApJ, 195, 137

Searle, L., \& Zinn, R. 1978, ApJ, 225, 357

Smith, G. H., \& Martell, S. 2003, PASP, 115, 1211

Smith, V. V., Cunha, K., Ivans, I. I., et al. 2005, ApJ, 633, 392

Soszynski, I., Udalski, A., Kubiak, M., et al. 2004, Acta Astron., 54, 129

Suntzeff, N. B., \& Kraft, R. P. 1996, AJ, 111, 1913

Ventura, P., D’Antona, F., Mazzitelli, I., \& Gratton, R. 2001, ApJ, 550, L65

Wheeler, J. C., Sneden, C., \& Truran, J. W. Jr. 1989, ARA\&A, 27, 279

Yong, D., Grundahl, F., Johnson, J. A., \& Asplund, M. 2008a, ApJ, 684, 1159

Yong, D., Melendez, J., Cunha, K., et al. 2008b, ApJ, 689, 1020

Zinn, R., \& West, M. J. 1984, ApJS, 5545

${ }^{6}$ Ter 7 is the most discrepant cluster in this comparison and is omitted from the fit. 